\title{
Test sequencing for sequential system diagnosis with precedence constraints and imperfect tests
}

\author{
Wenchao Wei*, Hongbo Li ${ }^{\dagger}$, Roel Leus ${ }^{\ddagger}$
}

\begin{abstract}
We study sequential system testing with the objective of minimizing the total expected testing costs. The goal is to discover the state of a system that consists of a set of independent components. The state of the system depends on the states of the individual components and is classified as working if at least a pre-specified number of components are working, otherwise it is said to be down. During the diagnostic testing procedure, components are tested one by one, in a pre-specified order. The resulting test sequencing problem is NP-hard with general precedence constraints even when the tests are perfect, in which case a component test always reports the correct state of the component. In this work, we will also consider the additional complication that tests can be imperfect, meaning that a test can report a component to be working when it is actually down, and vice versa. We develop a tabu search algorithm together with a simulation-based evaluation technique that incorporates importance sampling to find high-quality solutions within limited runtimes.
\end{abstract}

Keywords: system testing, sequencing and scheduling, precedence constraints, imperfect tests, tabu search, Monte Carlo simulation, importance sampling.

\section{Introduction}

System health monitoring for complex systems, such as a space shuttle, aircraft or integrated circuits, is crucial for reducing the likelihood of accidents due to sudden failures, and for improving system availability. It is also imperative that systems be tested before being put into operation, in order to ascertain their functionality. The problem of testing (diagnosing) a multi-component system through a series of tests of its components arises in many practical situations (Ünlüyurt, 2004): telecommunication (testing the connectivity of a network), manufacturing (quality control of newly manufactured products), electrical engineering (test whether a chip is functional or not), etc.

The $k$-out-of- $n$ configuration is a special case of a complex system that requires that, for the overall system to be functional, at least $k$ out of the total of $n$ components must be working. This

\footnotetext{
${ }^{*}$ School of Economics and Management, Beijing Jiaotong University, Beijing, China. E-mail: weiwenchao@bjtu.edu.cn.

${ }^{\dagger}$ School of Management, Shanghai University, Shanghai, China. E-mail: ishongboli@gmail.com.

†ORSTAT, Faculty of Economics and Business, KU Leuven, Leuven, Belgium. E-mail: Roel.Leus@kuleuven.be.
} 
configuration has a wide range of applications in both industrial and engineering systems (Ünlüyurt, 2004), such as a multi-display system in a cockpit, the multi-radiator system in a heating system, and the power grid of a city with excess power generators. Consider, for example, an airplane with four engines. Furthermore, suppose that the design of the aircraft is such that at least two engines are required to function for the aircraft to remain airborne. This means that the engines are related in a $k$-out-of- $n$ configuration, with $k=2$ and $n=4$. This is in literature sometimes also referred to as a "2-out-of-4:G" system, where $G$ means the system works or is "good"; a $k$-out-of- $n: G$ system is equivalent to an $(n-k+1)$-out-of- $n: F$ system, which fails (" $F$ ") if at least $(n-k+1)$ components fail. A so-called series system is an $n$-out-of- $n$ system and a parallel system is a 1-out-of- $n$ system.

In the context of system testing, the procedure of diagnosing a system consists of testing the components one by one in order to learn the state of the system. The same inspection procedure may be repeated thousands of times, and so it is important to minimize the total expected costs in the long run. The objective is to find a pre-determined test sequence such that the total expected costs are minimized. In practice, a system diagnosis typically also entails precedence requirements between the tests of some of the components, thus restricting the feasible component testing sequences, for instance when specific parts need to be removed from a machine first due to limited accessibility of some components or for guaranteeing the safety of dismantling of the system, or also when a certain test requires the output of other tests. In the related field of scheduling (Graham et al., 1979; Pinedo, 2012), precedence constraints have also been extensively used in modelling manufacturing process control and project planning.

We examine the case where individual component tests are imperfect, which means that a test can identify a component as working when in reality it is down, and vice versa; this can have severe implications. We focus on minimizing the expected cost to assess the state of the system with a specific confidence level. The references closest to our work are Nachlas et al. (1990), who focus only on series systems, and Wei et al. (2013), who study $k$-out-of- $n$ systems with perfect tests. To the best of our knowledge, however, the more general sequencing problem of imperfect tests for $k$-out-of- $n$ systems with precedence constraints has not yet been treated in the existing literature. It is the goal of this paper to fill exactly this gap.

The contributions of this work are threefold: (1) We describe a general model for sequential 
system diagnosis with precedence constraints and imperfect tests, which is a direct generalization of models that exist in the literature. (2) We develop an efficient method for finding good solutions. With general precedence constraints, minimum-cost sequential testing is NP-hard (Wei et al., 2013), and we will therefore resort to a tabu search (TS) algorithm for finding high-quality solutions for large instances. (3) We embed an importance sampling method into the TS procedure for evaluation of solutions.

The remainder of this paper is organized as follows: Section 2 provides a review of relevant literature. Definitions and a formal problem statement are given in Section 3. Section 4 describes importance sampling, and the overall TS algorithm is presented in Section 5. We report on our computational results in Section 6, and we round off with a summary and some conclusions in Section 7.

\section{Literature review}

An extensive literature review of different types of sequential testing problems can be found in Ünlüyurt (2004). Butterworth (1972) shows that the special cases of a parallel and a series system without precedence constraints are polynomially solvable. A polynomial-time algorithm for arbitrary $k$ was presented first by Salloum (1979), and independently by Ben-Dov (1981). With general precedence constraints, the testing problem for series systems is NP-hard (De Reyck \& Leus, 2008; Kelly, 1982). Computational results for sequencing tests of $k$-out-of- $n$ systems with general precedence constraints can be found in Wei et al. (2013). In this text, we will assume component tests to be independent; Cramer \& Kamps (1996) present another model in which the failure rate of the untested components is parametrically adjusted based on the number of preceding failures.

Most research efforts in the system-testing literature have been directed at finding testing policies for systems with $k=1$ and $k=n$ and with special precedence constraints (e.g., a series-parallel or a tree precedence graph). Relatively less attention has been given to imperfect testing, where due to a defective test design or inherent uncertainty, the outcome of a test only reflects the real condition of the component with a probability less than one, otherwise giving adverse information or even no information. Wei et al. (2017) study a polynomially solvable special case where the predictive error 
probabilities are the same for all the components (see Section 3.1 for definitions). The optimization of sequential search processes with imperfect tests has already been modeled and applied to various domains; we provide a brief survey below.

Imperfect testing has been introduced for so-called search problems. In the discrete search problem with a stationary target (Ahlswede \& Wegener, 1987), an item is assumed to be hidden in one of a set of boxes. Associated with each box $i$ is a prior probability $p_{i}\left(\right.$ with $\left.\sum_{i} p_{i}=1\right)$ that the item is hidden in that box and the overlook probability (type-II error) $a_{i}$ that the item will not be found in a particular search of that box even though the item is actually there. Value $a_{i}$ remains the same for every search of box $i$, and the time (cost) consumed in examining box $i$ is $c_{i}$. The search procedure does not stop before the item is found. Bellman (1957) was the first to describe an optimal policy to minimize the expected search cost by arranging the components in descending order of the ratio $p_{i}\left(1-a_{i}\right) / c_{i}$. Using this result, Gluss (1959) develops an optimal procedure for detecting the breakdown in a complex multi-component system. The stop criterion is the same: the fault can be concealed due to test errors, but is ultimately discovered by repeating tests until it is properly isolated. Wagner \& Davis (2001) extend the module concept proposed by Gluss (1959) for discrete sequential search and refer to it as a group activity.

The discrete search problem can also be seen as a representation of system testing. Besides the cost of testing, which depends on the set of components that are actually tested, Nachlas et al. (1990) also consider the consequences of a test error related to the disposition of the system after repair. If a false positive test result occurs, a functioning component is replaced and the failed component is left in place. If the system is then returned to service, the system fails immediately. If a false negative test result occurs, the overall test could indicate that no item fails; if the system is then returned to service, it might fail immediately and/or be scrapped.

\section{Problem description}

\subsection{Definitions}

We consider a system consisting of $n$ components, with component set $N=\{1,2, \ldots, n\}$. In order to discover the state of the system, the components will be tested sequentially on a single test machine. 
Table 1: Definitions of predictive errors, predictive values and type-I and type-II error positive predictive error $\varepsilon_{0 i}=\operatorname{Pr}\{$ component up $\mid$ outcome positive $\}$, negative predictive error $\varepsilon_{1 i}=\operatorname{Pr}\{$ component down $\mid$ outcome negative $\}$, positive predictive value $\left(1-\varepsilon_{0 i}\right)=\operatorname{Pr}\{$ component down $\mid$ outcome positive $\}$, negative predictive value $\left(1-\varepsilon_{1 i}\right)=\operatorname{Pr}\{$ component up $\mid$ outcome negative $\}$, type-I error $=\operatorname{Pr}\{$ outcome positive $\mid$ component up $\}$, type-II error $=\operatorname{Pr}\{$ outcome negative $\mid$ component down $\}$.

Each component is tested at most once (Nachlas et al., 1990, refer to this setting as "single-pass" testing). Each component is in one of two states: either working (up) or not working (down). The system functions (succeeds) if at least $k \leq n$ of the $n$ components are working and malfunctions (fails) if at least $(n-k+1)$ components are not working. We also refer the test of component $i$ simply as "test $i$." Throughout this paper, we will say that a positive test outcome is associated with the discovery of a failure. The outcome of test $i$ is a binary value $x_{i} \in \mathbb{B}=\{0,1\}$, where $x_{i}=0$ means that the test detects a fault (a positive outcome) and $x_{i}=1$ otherwise (a negative outcome). All outcomes are grouped into an $n$-dimensional binary vector $\mathbf{x}=\left(x_{1}, x_{2}, \ldots, x_{n}\right) \in \mathbb{B}^{n}$.

We study the situation where the measurements (tests) are imperfect: the outcome can be positive while the component is actually working, and vice versa. Imperfect testing can involve two types of test errors. The first type is false positive (type-I error), which means the tester concludes the component fails when really it is not; for example, a product can fail a quality test before being shipped, while in reality it is in good condition. The second type of error is false negative (typeII error), which means the outcome is negative but in reality the component fails, for example, a system check-up failing to detect the fault it was designed to find, in a computer system that really has the fault. Positive predictive value is the proportion of positive results that are truly positive, and negative predictive value is the proportion of negative results that are truly negative. Table 1 summarizes these definitions, where $\operatorname{Pr}\{A \mid B\}$ is the conditional probability of event $A$ knowing that event $B$ has occurred. Note that predictive values are conditioned on the test results and represent the probability of presence or absence of a fault in a given component test. For definitions of similar terms in diagnostic tests, we refer the reader to Altman \& Bland (1994a) and Altman \& Bland (1994b). 
Define $\chi_{i}$ as the probability that $x_{i}=1$, and $p_{i}$ the prior probability that component $i$ works. For ease of notation, we also define $q_{i}=1-p_{i}$ as the prior probability that component $i$ is down, and $\lambda_{i}=1-\chi_{i}$ the probability that $x_{i}=0$. Let $X_{i}$ represent the outcome of test $i$, which is a Bernoulli random variable with parameter $\chi_{i}$, and denote by $\mathbf{X}=\left(X_{1}, X_{2}, \ldots, X_{n}\right)$ the associated vector of random variables. The realization $x_{i}$ of $X_{i}$ is known only at the end of test $i$. We assume all variables $X_{i}$ to be mutually independent. It can be seen that $p_{i}=\left(1-\varepsilon_{1 i}\right) \chi_{i}+\varepsilon_{0 i}\left(1-\chi_{i}\right)$. Based on results obtained in Wei et al. (2017), we impose the natural condition that $\varepsilon_{0 i}+\varepsilon_{1 i}<1$, since otherwise the testing procedure is unable to provide meaningful information.

Consider a numerical example for testing one module of a specific product. Suppose the positive and negative predictive error are equal, $\varepsilon_{0}=\varepsilon_{1} \equiv \varepsilon=10 \%$, so if a test outcome is positive, we know that the module is not functional with probability 0.9 and is working with probability 0.1 ; a similar interpretation applies for the negative outcome. If $\chi=81.25 \%$ of the test outcomes of the production output for a given day is negative, then we can infer that the a-priori probability that a produced module works, is $0.1(1-\chi)+0.9 \chi=0.75$. The type-I error is computed as $0.1875 \times 0.1 / 0.75=0.025$ and the type-II error is $0.8125 \times 0.1 / 0.25=0.325$. In the foregoing example, we first fix the positive and negative predictive value and thereby also the complement $\varepsilon$, which represents the probability that a test outcome is wrong. The type-I and type-II errors then follow implicitly from this choice for $\varepsilon$ combined either with historical test data or with the a-priori probability that the module is functional. Alternatively, from given type-I and type-II errors the predictive errors could also be obtained.

A strict partial order relation $A$ on $N$ (a transitive and irreflexive binary relation) is also part of the input of a problem instance. Each $(i, j) \in A$ implies the precedence constraint that test $i$ should be conducted before test $j$. Figure 1 depicts a precedence graph for an example instance consisting of five components, with $N=\{1,2,3,4,5\}$, and the partial order relation $A$ containing the pairs $(1,3),(1,4),(1,5),(2,4),(2,5),(3,4)$ and $(3,5)$. Only the direct arcs are shown: the indirect arcs $(1,4)$ and $(1,5)$ are omitted from the figure. 


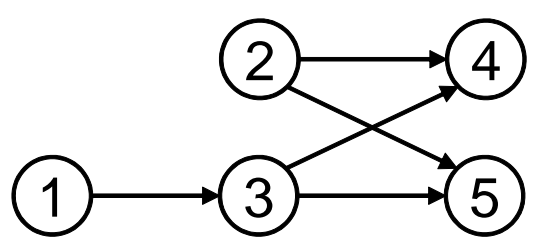

Figure 1: An example precedence graph

\subsection{Problem statement}

A solution to the sequencing problem under study is a testing policy, which decides how to proceed at each stage based on diagnosis information obtained from the preceding test outcomes. A test sequence or list $L=\left(l_{1}, l_{2}, \ldots, l_{n}\right)$ is a permutation of $N$. We call a sequence $L$ feasible if it respects the constraints in $A$, meaning that $(i, j) \in A \Rightarrow i$ appears before $j$ in $L$. Each feasible sequence defines a testing policy that successively schedules new tests in the order of the sequence. After each test, either a new component is selected or the diagnosis procedure is halted; this choice is based on the confidence level regarding the system's state.

Let $\theta_{1}(r, \mathbf{x}, L)$ be the probability that there are $k$ or more working components among the first $r$ tested ones following sequence $L$ and with outcome vector $\mathbf{x}$ (which would mean that the system functions), with $r \leq n$. Similarly, $\theta_{0}(r, \mathbf{x}, L)$ is the probability that there are at least $(n-k+1)$ failing components among the first $r$ tested components (leading to system malfunction). When there is no risk of confusion, we will use $\theta_{1}(r)$ and $\theta_{0}(r)$ for short. We define the overall system confidence level as $\theta(r)=\max \left\{\theta_{0}(r), \theta_{1}(r)\right\}$; in the context of isolating a single failure source, Raghavan et al. (1999) also use the term "level of confidence" to refer to this value. We study the optimization problem of designing a test policy with minimum expected total diagnostic costs. Given a specified threshold value $T$ on system confidence (e.g., $T=95 \%$ ), the inspection procedure stops when $\theta(r)$ reaches or exceeds $T$; if this stop criterion is never fulfilled then we perform all the $n$ tests (which maximizes the confidence of the result). The value of $T$ should follow from the requirements of the system or is specified by the user. In this paper, we focus on inspection policies that follow a fixed test sequence; such policies are called "elementary policies" in Wei et al. (2013).

Consider an example 3-out-of-5 system for a given outcome vector $(1,1,1,1,1)$ and test sequence $(1,2,3,4,5)$. With negative predictive error rates $\varepsilon_{11}=\varepsilon_{12}=\varepsilon_{13}=10 \%$ and $\varepsilon_{14}=\varepsilon_{15}=30 \%$ (the 
value of $\varepsilon_{0 i}$ is irrelevant here because all test outcomes are negative), we have $\theta_{1}(3)=72.9 \%$, $\theta_{1}(4)=87.48 \%$ and $\theta_{1}(5)=94.284 \%$, and also $\theta_{0}(3)=0.1 \%=0.1^{3}, \theta_{0}(4)=1.18 \%$ and $\theta_{0}(5)=$ $5.716 \%$, while $\theta_{0}(r)=\theta_{1}(r)=0$ for $r \leq 2$ (see Section 3.3 for details on the computation of these values). In case $T=90 \%$ then the diagnosis is not interrupted after the first four component tests because the obtained confidence level is not yet high enough, and so the fifth component is also tested; after this fifth test, $\theta_{1}(5) \geq T$ and so we conclude that the system is working. Had we worked with $T=85 \%$, however, then $\theta_{1}(4) \geq T$ and the fifth test would not have been necessary to achieve the (lower) desired confidence threshold. Value $T=85 \%$ would still have required four negative test outcomes although $k=3$ because $\theta_{1}(3)=0.729<0.85$. For $T=95 \%$, even after all five component tests the threshold is not attained, and so we halt without firm conclusion; we will call such a diagnosis inconclusive.

Given a test sequence, the tester follows the sequence and test the components one by one until one of the stop criteria is met: either the threshold confidence level is achieved, or all the components have been tested. For a given outcome vector $\mathbf{x}$, the actually tested series of components contains a subset of $N$, namely the (partial) job list $L \mid \mathbf{x}$ obtained when $L$ is truncated as soon as a stopping criterion is reached. We call this executed subsequence a schedule. Let value $c_{i}$ represent the cost of test $i$, for each $i \in N$. The corresponding cost of schedule $L \mid \mathbf{x}$ is then $f(L, \mathbf{x})=\sum_{t=1}^{|L| \mathbf{x} \mid} c_{L(t)}$, where $L(t)$ is the $t$-th element of $L$. Our objective is to find a sequence that minimizes the following expression, which represents the expected cost of (the policy defined by) sequence $L$ :

$$
\mathbb{E}[f(L, \mathbf{X})]=\sum_{\mathbf{x} \in \mathbb{B}^{n}}\left(\prod_{i: x_{i}=1} \chi_{i}\right)\left(\prod_{i: x_{i}=0} \lambda_{i}\right) f(L, \mathbf{x}),
$$

with $\mathbb{E}[\cdot]$ the expectation operator with respect to $\mathbf{X}$. Note that testing the components sequentially is a dominant decision for this objective function, but this does not necessarily apply for other "classic" scheduling objectives such as makespan minimization.

\subsection{Computing the system confidence level}

We define $Q^{(\mathbf{x}, L)}(w, r)$ as the probability that there are exactly $w$ components working among the first $r$ components tested (consequently, $r-w$ components are down) according to sequence $L$ 
and outcome $\mathbf{x}$; below we use $Q(w, r)$ for short. We initialize $Q(0,0)=1$, and $Q(w, r)=0$ when $w=-1$ or $w>r \geq 0$, and we propose the following recursive formula to generate each $Q(w, r)$ for $w=0,1, \ldots, n$ and $r=1,2, \ldots, n$, with $w \leq r:$

$$
\begin{aligned}
Q(w, r)= & Q(w, r-1)\left(x_{L(r)} \varepsilon_{1 L(r)}+\left(1-x_{L(r)}\right)\left(1-\varepsilon_{0 L(r)}\right)\right) \\
& +Q(w-1, r-1)\left(x_{L(r)}\left(1-\varepsilon_{1 L(r)}\right)+\left(1-x_{L(r)}\right) \varepsilon_{0 L(r)}\right) .
\end{aligned}
$$

The remaining values $Q(w, r)$ are set to zero. Intuitively, to have $w$ working components after $r$ tests, either there were already $w$ working components after $r-1$ tests (which corresponds with the first term in the right-hand side), and then the last test $L(r)$ pertains to a failing component, which is either correctly observed $\left(x_{L(r)}=0\right)$ with probability $1-\varepsilon_{0 L(r)}$, or wrongly observed $\left(x_{L(r)}=1\right)$ with probability $\varepsilon_{1 L(r)}$. Alternatively, there were only $w-1$ working components after the first $r-1$ tests, and then a similar reasoning can be followed to obtain the second term in the right-hand side.

Table 2 illustrates the computation of $Q(w, r)$ for a system with $n=4$, for a given outcome $\mathbf{x}=(1,0,1,0)$ and sequence $L=(1,2,3,4)$. Computing all values $Q(w, r)$ requires $O\left(n^{2}\right)$ operations in total for a given sequence and outcome vector. Given the values of $Q(w, r)$, we derive $\theta_{1}(r)$ and $\theta_{0}(r)$ as follows:

$$
\begin{aligned}
& \theta_{1}(r)= \begin{cases}0, & \text { if } r<k, \\
\sum_{w=k}^{r} Q(w, r), & \text { if } r \geq k .\end{cases} \\
& \theta_{0}(r)= \begin{cases}0, & \text { if } r<n-k+1, \\
\sum_{w=0}^{r-n+k-1} Q(w, r), & \text { if } r \geq n-k+1 .\end{cases}
\end{aligned}
$$

The value of $\theta_{1}$ (4) computed in Section 3.2 for the 3-out-of-5 instance with outcome vector $(1,1,1,1,1)$, for instance, can be obtained as $Q(3,4)+Q(4,4)=0.8748$. Obviously, if the positive predictive errors are the same for all tests $\left(\varepsilon_{0 i} \equiv \varepsilon_{0}\right)$ and all negative errors are the same $\left(\varepsilon_{1 i} \equiv \varepsilon_{1}\right)$ then the expressions in the table can be significantly simplified (see Wei et al., 2017). 
Table 2: Illustration of the computation of the values of $Q(w, r)$ for outcome $\mathbf{x}=(1,0,1,0)$ and sequence $L=(1,2,3,4)$. Some table entries are not included for lack of space, these have been replaced by "..."

\begin{tabular}{|c|c|c|c|c|}
\hline $\begin{aligned} r=L(r) & = \\
x_{L(r)} & =\end{aligned}$ & $\begin{array}{l}1 \\
1\end{array}$ & $\begin{array}{l}2 \\
0\end{array}$ & $\begin{array}{l}3 \\
1\end{array}$ & $\begin{array}{l}4 \\
0\end{array}$ \\
\hline$w=0$ & $\varepsilon_{11}$ & $\varepsilon_{11}\left(1-\varepsilon_{02}\right)$ & $\varepsilon_{13} \varepsilon_{11}\left(1-\varepsilon_{02}\right)$ & $\varepsilon_{13} \varepsilon_{11}\left(1-\varepsilon_{02}\right)\left(1-\varepsilon_{04}\right)$ \\
\hline$w=1$ & $1-\varepsilon_{11}$ & $\begin{array}{r}\varepsilon_{02} \varepsilon_{11} \\
+\left(1-\varepsilon_{02}\right)\left(1-\varepsilon_{11}\right)\end{array}$ & $\begin{array}{r}\varepsilon_{11}\left(1-\varepsilon_{13}\right)\left(1-\varepsilon_{02}\right) \\
+\varepsilon_{02} \varepsilon_{11} \varepsilon_{13} \\
+\varepsilon_{13}\left(1-\varepsilon_{02}\right)\left(1-\varepsilon_{11}\right)\end{array}$ & \\
\hline$w=2$ & 0 & $\varepsilon_{02}\left(1-\varepsilon_{11}\right)$ & $\begin{array}{r}\varepsilon_{02} \varepsilon_{11}\left(1-\varepsilon_{13}\right) \\
+\left(1-\varepsilon_{02}\right)\left(1-\varepsilon_{11}\right)\left(1-\varepsilon_{13}\right) \\
+\varepsilon_{02} \varepsilon_{13}\left(1-\varepsilon_{11}\right) \\
\end{array}$ & $\cdots$ \\
\hline$w=3$ & 0 & 0 & $\varepsilon_{02}\left(1-\varepsilon_{11}\right)\left(1-\varepsilon_{13}\right)$ & $\cdots$ \\
\hline$w=4$ & 0 & 0 & 0 & $\varepsilon_{02} \varepsilon_{04}\left(1-\varepsilon_{11}\right)\left(1-\varepsilon_{13}\right)$ \\
\hline$\sum_{w=0}^{4} Q(w, r)=$ & 1 & 1 & 1 & 1 \\
\hline
\end{tabular}

\section{Solution evaluation with importance sampling}

\subsection{Solution evaluation using simulation}

Although the evaluation of a test sequence can be done efficiently for a given outcome vector (see Section 3.3), the overall evaluation of the total expected testing costs for a given sequence is still quite time-consuming due to the exponential number of possible outcomes. We therefore approximate the objective function (1) by means of Monte Carlo simulation. A silimar approach has been followed in the context of system reliability evaluation (Huseby et al., 2004) and stochastic resource-constrained scheduling (Ballestín \& Leus, 2009; Stork, 2001). An approximation of the expected cost of a list $L$ is based on sampling a number of realizations $\mathbf{x}$ from $\mathbb{B}^{n}$, which are gathered in $\overline{\mathbb{B}}^{n} \subset \mathbb{B}^{n}$. If $M=\left|\overline{\mathbb{B}}^{n}\right|$ then for a solution defined by $L$, the objective is approximated as follows:

$$
\mathbb{E}[f(L, \mathbf{X})] \approx \frac{1}{M} \sum_{\mathbf{x} \in \mathbb{B}^{n}} f(L, \mathbf{x})
$$

The calculation of the step-wise accuracies for a given outcome needs $O\left(n^{2}\right)$ time (see Section 3.3), and so does the cost function $f(\cdot)$. The approximation in Equation (5) can thus be computed in $O\left(M n^{2}\right)$ time. 


\subsection{Variance reduction techniques}

The literature on sampling from discrete distributions, e.g., the multivariate Bernoulli distribution, is less extensive than for continuous distributions. The problem of sampling from multivariate Bernoulli sample spaces has been studied by Biller \& Ghosh (2006) and recently by Schäfer \& Chopin (2013). Of special relevance to this paper is Broström \& Nilsson (2000), who study the problem of sampling independent Bernoulli variables given their sum; see also Nilsson (1997). Huseby et al. (2004) also adopt a similar methodology which they call conditional Monte Carlo simulation to evaluate the system reliability: they group the simulations based on the sum of all the $n$ binary variables $x_{i}$, and each simulation from the same group renders the same system state. They claim that 10 simulations produce nearly perfect results for a five-component system; this corresponds with $10 / 32 \approx 31 \%$ of the total number of scenarios. We will use a similar approach.

It is well known that rather than simple random sampling of $M$ observations from $\mathbb{B}^{n}$, a number of variance reduction techniques can be applied to increase the precision of the estimate by means of a more judicious choice of the $M$ observations (cfr. Law \& Kelton, 2000). Conditional Monte Carlo simulation as described above is a particular form of stratified sampling (see Bratley et al., 1983 ), where $\mathbb{B}^{n}$ is partitioned into a number of subsets before sampling, and then simple random sampling is applied within each subset. With stratification, $\mathbb{B}^{n}$ is partitioned into $n+1$ groups $B_{0}, B_{1}, \ldots, B_{n}$, where $B_{\sigma}$ gathers all the realizations $\mathbf{x}$ that have $\sum_{i=1}^{n} x_{i}=\sigma$. Let $V=\sum_{i=1}^{n} X_{i} ; V$ is a Binomial random variable if all $\chi_{i}$ are identical, but this is not the case in general. We then sample $M_{\sigma}$ observations from $B_{\sigma}$, with $\sum_{\sigma=0}^{n} M_{\sigma}=M$. In stratified sampling,

$$
M_{\sigma} \approx M \cdot \operatorname{Pr}\left\{\sum_{j=1}^{n} x_{j}=\sigma\right\}
$$

and sampling from $B_{\sigma}$ is performed following the conditional distribution of $\mathbf{X}$ given that $V=\sigma$. To calculate the sampling distribution of $\mathbf{X}$, we need the following partial sums:

$$
V_{j}=\sum_{i=j}^{n} X_{i}, \quad j=1, \ldots, n
$$


With $V_{n} \equiv X_{n}$, we calculate the distribution of $V_{1}, \ldots, V_{n-1}$ recursively using the following formula:

$$
\operatorname{Pr}\left\{V_{j}=m\right\}=\chi_{j} \operatorname{Pr}\left\{V_{j+1}=m-1\right\}+\lambda_{j} \operatorname{Pr}\left\{V_{j+1}=m\right\}
$$

for $j=n-1, n-2, \ldots, 1$ and $m \in\{0,1, \ldots, n-j+1\}$, where obviously $\operatorname{Pr}\left\{V_{i}=-1\right\}=0$ for all $i$.

For a given value of $\sigma \in\{0,1, \ldots, n\}$, we start out by sampling $X_{1}$ conditional on $V=\sigma$. We then continue by sampling $X_{2}$ conditional on $V=\sigma$ and $X_{1}=x_{1}$, where $x_{1}$ is the sampled outcome of $X_{1}$ (either 1 or 0 ), and so on. Specifically, the sampling probability of $X_{j}$ with $j>1$ is

$$
\begin{aligned}
\operatorname{Pr}\left\{X_{j}=x_{j} \mid X_{1}=x_{1}, \ldots, X_{j-1}=x_{j-1}, V=\sigma\right\} & =\frac{\operatorname{Pr}\left\{X_{j}=x_{j}, V=\sigma \mid X_{1}=x_{1}, \ldots, X_{j-1}=x_{j-1}\right\}}{\operatorname{Pr}\left\{V=\sigma \mid X_{1}=x_{1}, \ldots, X_{j-1}=x_{j-1}\right\}} \\
& =\frac{\operatorname{Pr}\left\{X_{j}=x_{j}, V_{j+1}=\sigma-\sum_{m=1}^{j} x_{m}\right\}}{\operatorname{Pr}\left\{V_{j}=\sigma-\sum_{m=1}^{j-1} x_{m}\right\}} \\
& =\frac{\chi_{j}^{x_{j}} \lambda_{j}^{1-x_{j}} \operatorname{Pr}\left\{V_{j+1}=\sigma-\sum_{m=1}^{j} x_{m}\right\}}{\operatorname{Pr}\left\{V_{j}=\sigma-\sum_{m=1}^{j-1} x_{m}\right\}} .
\end{aligned}
$$

The distributions of the $V_{j}$ are computed before running the simulation, and only the conditional probabilities needed along the way during the simulation are calculated. Therefore, in each simulation run $n$ probabilities are calculated, and this can be done in $O(n)$ time.

Importance sampling is another variance reduction technique that generalizes stratified sampling (Bratley et al., 1983). The idea behind importance sampling is that certain values of the input random variables in a simulation have more impact on the parameter being estimated than others, and the sample sizes $M_{\sigma}$ for groups $B_{\sigma}$ containing many of these variables will be chosen higher than as suggested by Equation (6). This use of "biased" distributions will result in a biased estimator if it is applied directly in the simulation, so the simulation outputs should be weighted to ensure that the estimator is unbiased. Put differently, different observations might receive different weights to remove bias (Bratley et al., 1983). 


\subsection{Our implementation}

To the best of our knowledge, we are the first in the field of sequencing and scheduling to adopt importance sampling for solution evaluation. We motivate our approach based on Figure 2. For one particular scheduling instance ${ }^{1}$ with $n=10$, there are 1024 different realizations of $\mathbf{X}$ in total, which can be partitioned into 11 groups. The probabilities of occurrence of each of these groups $B_{0}, B_{1}, \ldots, B_{10}$ is shown in Figure 2(a). In total, there are 7900 lists that constitute a linear extension of the input precedence graph. In the three subfigures 2(b), 2(c) and 2(d), we report the maximum difference in expected cost between any two solutions (lists), with the expected cost computed conditional on a specific choice for $V$ (which is on the horizontal axis). This is examined for $k=2,5$ and 8 . We observe that the maximum cost difference is different between different values of $V$, and also that its highest value is obtained around $V=k$ (for $k=8$ the highest value is obtained for $V=7)$.

The main purpose of our sampling-based evaluation is to distinguish between good and bad lists, so to distinguish between low and high testing cost. If for a pair of solutions the costs under two realizations are the same then these realizations should not both be used, because two realizations then do not bring along more information than a single one. This is our motivation for possibly sampling less scenarios than prescribed by Equation (6) especially from those subsets $B_{j}$ where the cost function is relatively flat, and more from $B_{j}$ for $j$-values that have a high score in Figures 2(b), 2(c) and 2(d). Concretely, we will sample the following number of observations from subset $B_{\sigma}$ $(\sigma=0,1, \ldots, n)$ :

$$
M_{\sigma}=M \cdot \frac{\operatorname{Pr}\{V=\sigma\} \cdot D_{\sigma}^{\gamma}}{\sum_{\sigma=0}^{n} \operatorname{Pr}\{V=\sigma\} \cdot D_{\sigma}^{\gamma}} .
$$

The value $D_{\sigma}$ is an approximation of the maximum cost difference for given $\sigma$, and the power $\gamma$ allows to tune the importance of this factor (value $\gamma=0$ leads to the stratified sampling method in Equation (6)). Note that for general $n$, the calculation of these cost differences is still timeconsuming and should indeed be approximated due to the huge number of solutions and scenarios (although for $n=10$ we could still do it in an exact fashion for the example in Figure 2, but at

\footnotetext{
${ }^{1}$ The instance $s \_n 10 \_o s 4 \_1$, with order strength $O S=0.4$ (see Section 6.1 for more details on the instance sets and their parameters)
} 


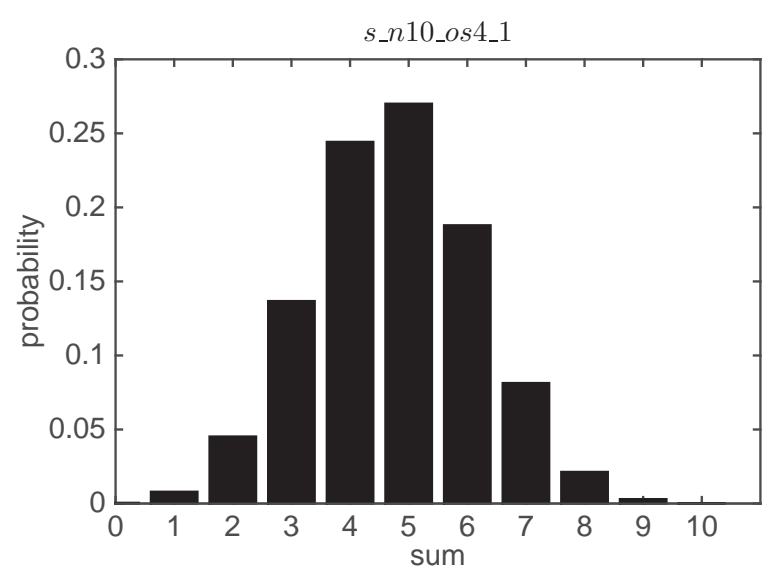

(a) Occurrence probabilities

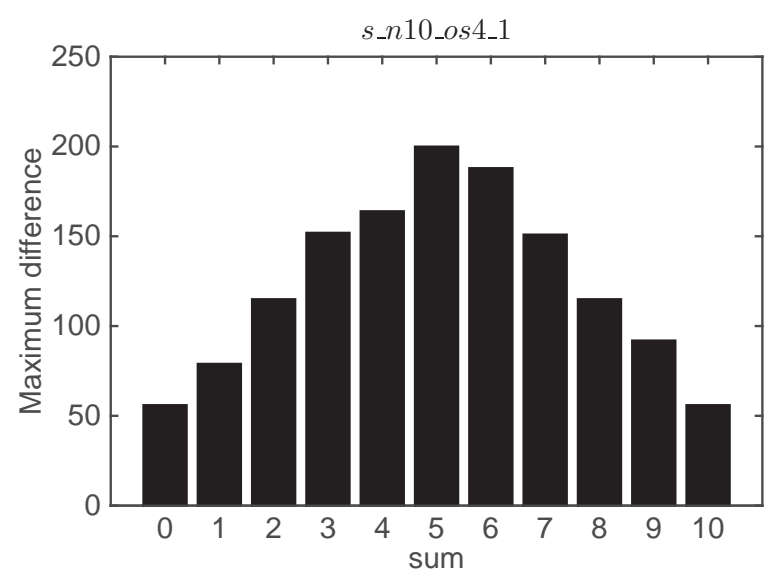

(c) $k=5$

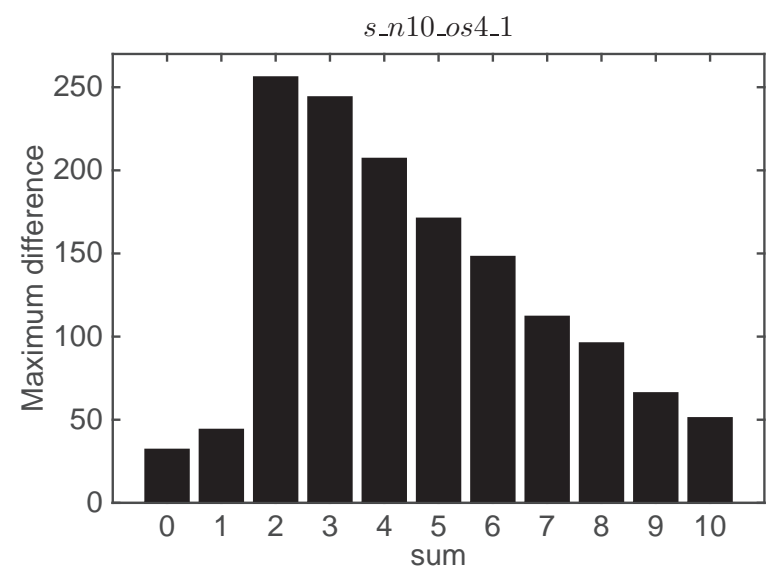

(b) $k=2$

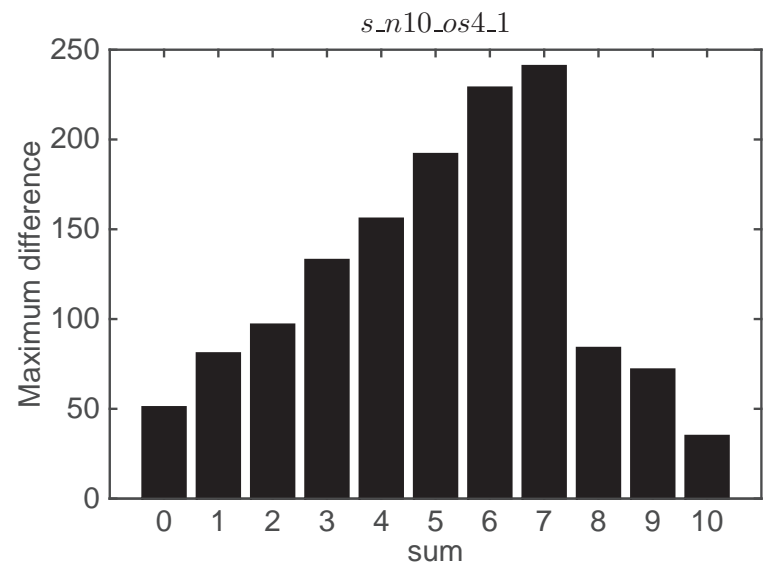

(d) $k=8$

Figure 2: Occurrence probabilities, and the maximum cost difference over all feasible lists, conditional on the sum of the variables

the expense of $7900 \times 1024$ runs of the $O\left(n^{2}\right)$ evaluation algorithm). We construct list $L_{\min }=$ $\left\{l_{1}^{\min }, l_{2}^{\min }, \ldots, l_{n}^{\min }\right\}$ by greedily appending the components one by one, where the next component to be included is one with lowest testing cost whose predecessors have all be included already. Similarly, list $L_{\max }=\left\{l_{1}^{\max }, l_{2}^{\max }, \ldots, l_{n}^{\max }\right\}$ is a linear extension of the input order that is close to a high-to-low-cost ordering of the components. Then for realizations in group $\sigma<k$, the least total testing cost is estimated by $\sum_{j=1}^{n-k+1} c_{l_{j}^{\min }}$, and the highest total testing cost is estimated by $\sum_{j=1}^{n-k+1+\sigma} c_{l_{j}^{\max }}$. In this way, the maximum cost difference can be approximated by the difference between the foregoing two values, and a similar approach can be followed for groups $\sigma \geq k$, leading 
to

$$
D_{\sigma}= \begin{cases}\sum_{j=1}^{n-k+1+\sigma} c_{l_{j}^{\max }}-\sum_{j=1}^{n-k+1} c_{l_{j}^{\min }}, & \forall \sigma<k, \\ \sum_{j=1}^{n-\sigma+k} c_{l_{j}^{\max }}-\sum_{j=1}^{k} c_{l_{j}^{\min }}, & \forall \sigma \geq k .\end{cases}
$$

Subsequently, the group averages $M_{\sigma}$ for the different groups $B_{\sigma}(\sigma \in\{0,1, \ldots, n\})$ are combined in a weighted average with weights $\operatorname{Pr}\{V=\sigma\}$ to produce an unbiased estimator for $\mathbb{E}[f(L, \mathbf{X})]$.

\section{Tabu search}

\subsection{General framework}

As mentioned previously, the problem at hand is NP-hard, and we therefore resort to a heuristic method for its resolution. A solution representation as a sequence allows for easy manipulation by a meta-heuristic search procedure. In this paper, we opt for developing a tabu search (TS) procedure. TS (Glover \& Laguna, 1997) is a meta-heuristic framework that directs a local search procedure to an exploration of the solution space beyond local optimality. It has been extensively used to solve sequencing and scheduling problems, including single machine scheduling (Bilge et al., 2007; Laguna et al., 1991; Wan \& Yen, 2002), job shop scheduling (Petrovic et al., 2007), car sequencing (Cordeau et al., 2008), the binary assignment problem (Kochenberger et al., 2002), the production positioning problem (Luo et al., 2012), and so on. Many other meta-heuristic frameworks, such as simulated annealing and tabu search, exist and could be plugged in. Quite some references show that, dependent on the particular problem under study, TS tends to yield superior performance (Arostegui et al., 2006; Augugliaro et al., 1999; Morton \& Pentico, 1993). Other references point out that it does not really make a lot of sense to ask general questions such as "Is tabu search better than simulated annealing?" (Sörensen, 2015), and underline the importance of more fundamental generic choices such as neighourhood generation (Hertz \& Widmer, 2003).

The basic functioning of TS can be summarized as follows. Starting from an initial feasible solution, this local search procedure iteratively moves from the current solution to its best neighbour, which is a new solution that is obtained from the current one via minor modifications. This move is made even if the new solution is not better than the current one, and this until a stopping criterion is 
satisfied. Dependent on the definition of the neighbourhood, exhaustively scanning each neighbour may be prohibitively expensive in terms of runtime, and developing a restricted candidate list of promising neighbours (moves) may be helpful.

In order to avoid cycling and getting stuck in suboptimal regions of the search space, moves that lead back to previously explored regions can be forbidden or classified as tabu, forming the shortterm memory also referred to as tabu list. If a potential move appears on the tabu list, it cannot be executed, unless it leads to a new best solution. The length of time during which a certain move is classified as tabu and thus appears in the tabu list (the tabu tenure), is an important parameter for tabu search. This tabu tenure can be kept constant or be varied dynamically throughout the search procedure. TS does not always succeed in directing the search into regions where the best solutions are found by solely employing short-term memory strategies. Long-term memory strategies that drive the search into new regions of the solution space (diversification) or that stimulate a more thorough examination in some good or promising regions (intensification) may be needed.

For each solution evaluation, we use importance sampling as described in Section 4, in which for each sampled scenario we calculate the step-wise accuracies and the cost function following Section 3.2 and 3.3. Below we describe how the key generic aspects of the TS algorithm are tailored to finding high-quality solutions for $k$-out- $n$ testing with imperfect tests and precedence constraints.

\subsection{Key algorithmic details}

\subsubsection{Initial solution generation}

A solution is gradually built by selecting components from $N$ and appending them to the end of a partial list. At each iteration, only a subset of $N$ is considered, only containing the components whose predecessors have already been included in the list. One component with lowest ratio $c_{i} / r_{i}$ is selected at each step, where $r_{i}=\chi_{i}\left(1-\varepsilon_{0 i}\right)+\left(1-\chi_{i}\right) \varepsilon_{1 i}$ if $k<\lfloor n / 2\rfloor$, and $r_{i}=\left(1-\chi_{i}\right)(1-$ $\left.\varepsilon_{1 i}\right)+\chi_{i} \varepsilon_{0 i}$ otherwise; we refer to this heuristic procedure as HR. A possible alternative uses more information on the structure of the precedence graph by selecting one element with the highest number of (direct and indirect) successors at each step; this rule is referred to as HP. 


\subsubsection{Neighbourhood generation}

Insert moves and pairwise exchanges (swaps) are frequently used move types for neighbourhood generation in sequencing problems. An insert move insert $\left(l_{i}, l_{j}\right)$ identifies two jobs $l_{i}$ and $l_{j}(i<j)$ in the current sequence $L=\left(l_{1}, l_{2}, \ldots, l_{n}\right)$ and inserts job $l_{i}$ between $l_{j}$ and its direct successor in the list. A swap move exchanges the location of two jobs so that each job is placed in the location previously occupied by the other. A swap move can be considered as a combination of two insert moves.

In the following, we will further examine the insert moves and pairwise exchanges when precedence constraints are considered. Clearly, a test cannot be scheduled before its predecessors or after its successors. In any feasible sequence, if $i<j$, then for all $i<r \leq j,\left(l_{r}, l_{i}\right) \notin A$ holds. An insert move $\operatorname{insert}\left(l_{i}, l_{j}\right)$ for $i<j$ inserts the test currently at position $i$ in between position $j$ and its direct successor in the list. Such a move must respect the precedence constraints $A$ as well, otherwise it can not be performed. Specifically, this necessitates $\left(l_{i}, l_{r}\right) \notin A$ for all $i<r \leq j$. Denote $F_{i}=\{j \in N \mid(i, j) \notin A \wedge(j, i) \notin A\}$ as the set of tests that are not precedence-related with test $i$. We say move $\operatorname{insert}\left(l_{i}, l_{j}\right)$ with $i<j$ is feasible if and only if $l_{r} \in F_{l_{i}}$ for $i<r \leq j$. Analogously, a move insert $\left(l_{i}, l_{j}\right)$ for which $i>j$ inserts the test currently at position $i$ between the test at position $j$ and its direct predecessor in the list; its feasibility requires $l_{r} \in F_{l_{i}}$ for $j \leq r<i$.

A swap move $\operatorname{swap}\left(l_{i}, l_{j}\right)$ with $i<j$ will be considered only when both $l_{k} \in F_{l_{i}}$ and $l_{k} \in F_{l_{j}}$ hold for all $i<k<j$. Move $\operatorname{swap}\left(l_{i}, l_{j}\right)$ equates with performing $\operatorname{insert}\left(l_{j}, l_{i}\right)$ and $\operatorname{insert}\left(l_{i+1}, l_{j}\right)$ consecutively. It should be noted that although $\operatorname{swap}\left(l_{i}, l_{j}\right)$ is equivalent to $\operatorname{swap}\left(l_{j}, l_{i}\right)$, the same is not the case for insert $\left(l_{i}, l_{j}\right)$. For instance, in the case of the insert $\left(l_{i}, l_{j}\right)(i<j)$, position $i$ (which is component 1 at position 2 in Figure 3) contains the component that is displaced to make room for the components that are to be shifted to the left. The neighbourhood used in this study is a hybrid one that consists of the complete insert neighbourhood and the complete swap neighbourhood.

The procedure described in Section 4 is used to evaluate the neighbourhood solutions. Furthermore, it is easy to verify that, for a given outcome, only recalculating the accuracies for the components whose positions have been changed in the current move is necessary. In Table 2, for instance, exchanging components 1 and 2 still leads to the same values in columns 3 and 4 . 


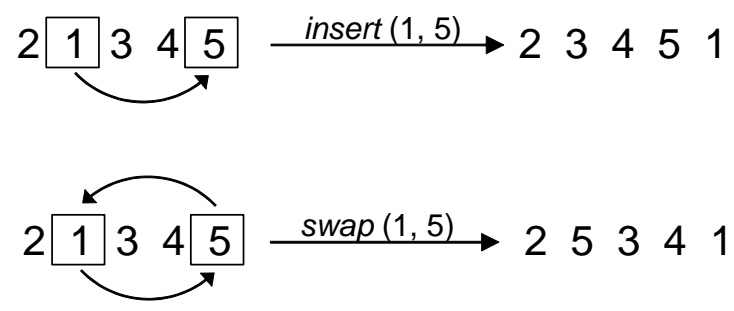

Figure 3: The insert move and swap move

\subsubsection{Tabu list}

A chief mechanism for exploiting memory in TS is to classify a subset of the moves in a neighbourhood as forbidden (tabu). The classification depends on the history of the search, particularly manifested in the recency or frequency with which a certain move or solution components, called attributes, have participated in generating past solutions. Some choices of attributes may be better than others (Glover \& Laguna, 1997). In its simplest form, a tabu list is a short-term set of the solutions that have been visited in the recent past. A potential solution cannot be revisited until the corresponding move is removed from the tabu list. Possible classifications are:

- Move-based tabu. A move is made tabu active after it is performed, and this status is maintained during the tabu tenure. For example, the move in Figure 3 leads to $(1,5)$ being tabu active. Clearly, this move-based tabu list only needs a single check for each candidate move. An $n \times n$ matrix tabu can be used to implement this short-term memory for each move type. This matrix is initialized with zeros, and whenever an insert $(i, j)$ is implemented we set $\operatorname{tabu}(i, j)$ to the current iteration counter plus the tabu tenure during which the move will be non-admissible. The swap moves are handled separately in another matrix.

- Position-based tabu. The position-based tabu classification aims to prohibit some of the positional relationships that were broken by recent moves from being constructed again during tabu tenure. It can be summarized as follows: after a move insert $\left(l_{i}, l_{j}\right)$, all moves (both insert and swap) resulting in permutations in which the two tests in the pairs $\left(l_{j}, l_{j+1}\right),\left(l_{i}, l_{i+1}\right)$ and $\left(l_{i-1}, l_{i}\right)$ appear as two consecutive tests, are classified as tabu. After $\operatorname{swap}\left(l_{i}, l_{j}\right)$, the four pairs $\left(l_{j-1}, l_{j}\right),\left(l_{j}, l_{j+1}\right),\left(l_{i-1}, l_{i}\right)$ and $\left(l_{i}, l_{i+1}\right)$ become tabu active. The swap move in Figure 3 , for example, leads to the pairs $(2,1),(1,3)$ and $(4,5)$ becoming tabu active. 
The position-based tabu classification contains more information about an already visited solution and is generally considered to be more effective in preventing cycling, compared to move-based tabu classifications (Glover \& Laguna, 1997). This is the case for our problem as well, as verified by our preliminary experiments. The tabu status of a move is overridden if it yields a solution better than the best obtained so far. In other words, we apply the best move found that is not tabu, unless a tabu move leads to an improved upper bound. The executed move is subsequently made tabu active.

As pointed out in Section 5.2.2, since a move may violate the precedence constraints, not every insert or swap move is allowed. Actually, only a limited number of insert moves are considered for each component. As an example, consider the current solution $(1,2,3,4,5)$ with precedence graph as depicted in Figure 1. For component 2, the only feasible insert moves are insert $(2,4)$ and insert $(2,3)$. Additionally, in some rare cases, e.g., instances with very dense precedence graph and long tabu tenure, at some point during the search procedure there may be no move to proceed. If this occurs then we empty the tabu list and continue the search from the current solution.

\subsubsection{Candidate list strategy}

The exploration of a neighbourhood proceeds as follows: first we generate a candidate list of possible moves and then we evaluate each one until an acceptance criterion is met. A review of more sophisticated strategies is given in Glover \& Laguna (1997). Possible techniques are first ascent (FA), which takes the first improvement in quality found, and steepest ascent (SA), which systematically examines the entire list and accepts the most improving move available. Steepest ascent is less attractive especially when the neighbourhoods to be evaluated become quite large; the size increases with $O\left(n^{2}\right)$.

An alternative method is to use a candidate list strategy that selects a subset of the eligible moves for evaluation. A simple approach is to construct this subset at random (Reeves, 1993). Another option is to perform moves only on adjacent components (AC), e.g., insert a component immediately after its direct successor or before its direct predecessor in the list. A logical extension is to restrict to moves that pertain to positions that are not too far apart in the permutation. In this study, we call AC1 the candidate list strategy that only considers moves between the current 
component and its direct successor and predecessor in the list; AC2 allows moves performed with the first two successors and predecessors in the list; and so on.

Candidate list strategies can also be developed based on problem-specific characteristics; these are often called move heuristics and apply dominance rules that retain only a number of promising moves, or exclude moves that are less likely to improve the objective, where the retention or exclusion is usually heuristic in nature. In Section 6 we will also examine the performance of the ratio heuristic (RH), which only allows moves of components $l_{i}, l_{j}$ when the inequality $c_{j} / r_{j}>c_{i} / r_{i}$ is satisfied after the move (see Section 5.2.1 for the definition of $r_{i}$ ).

\subsubsection{Tabu tenure}

There are two main approaches to tenure selection: either using a single tenure value throughout the entire search, or dynamically varying the tenure as the search progresses.

To decide the single tenure value, we first determine a range of tenure values that promise a good solution quality for each instance, and then select a fixed tenure value based on the attributes of the instance (i.e., instance size, system configuration, density of the precedence constraints).

A dynamic tenure strategy applies a sequence of different tenure values within a pre-defined range and repeats this sequence throughout the search. Systematically varying the tenure values in this manner provides a balance between search intensification and diversification. Short tabu tenures allow an extensive search of the neighbourhood, while long tenures direct the search to a different part of the solution space (Glover \& Laguna, 1997).

We note that due to the system configuration and the stop criteria, there exist indifferent moves that neither improve nor worsen the current solution. Specifically, a swap or insert move within the first $\min \{k, n-k+1\}$ components leads to a solution with the same objective value as the current one. The number of such moves grows fast as $k$ is closer to $n / 2$. This type of move makes it easier for the search to become trapped in a local optimum. Selecting a large tenure value for such moves or excluding them from the search procedure is helpful. 


\subsubsection{Long-term strategies}

Besides the dynamic tabu tenure strategy introduced above as a compromise between intensification and diversification, some further methods are developed as long-term strategies.

Diversification strategies efficiently employ the knowledge of the search history and progressively guide the search towards new regions of the solution space. In our algorithm, the search history is reflected in a large tenure, which is also the main instrument to move the search out of the current region. A similar strategy is also used in Bilge et al. (2004) for a single machine scheduling problem. We achieve diversification by multiplying the current tenure value by a large multiplier after a pre-specified number of non-improving iterations.

Our intensification strategy consists in storing a certain number of elite solutions during the short-term TS and then restarting and carrying out a search for a given time period, in our case one minute, on each of these solutions. An elite solution is defined as a solution that can survive as the best-so-far for a given number of iterations.

\section{Computational results}

In this section, we present a summary of the computational experiments with our implementation of the TS algorithm for sequential system testing with imperfect tests, which was developed in the previous sections. The details of our experimental setup are described in Section 6.1. The TS parameters are finetuned through extensive experiments, on which we report in Section 6.2. A detailed analysis of the algorithmic performance is provided in Section 6.3.

\subsection{Experimental setup}

The algorithm is coded in C++ using Microsoft Visual Studio 2010. The experiments are run on a Dell Laptop Latitude E6400 with an Intel Core 2 2.66-GHz processor and 4 GB RAM, equipped with Windows 7 Enterprise Service Pack 1. We use the instances with perfect tests from Wei et al. (2013) as our test bed. For various values for the number $n$ of components, we consider three values $0.4,0.6$ and 0.8 for the order strength $O S$, which is a measure of network density, computed as the number of comparable component pairs divided by the maximum number $n(n-1) / 2$ of such 
pairs. The (integer) component testing costs are generated as independent realizations of a discrete uniform random variable on $[0,50]$ and the success probabilities are chosen randomly from range $[0.2,0.8]$, leading to 10 instances for each combination of $O S$ and $n$. The error probabilities $\varepsilon_{i 0}$ and $\varepsilon_{i 1}$ are drawn from the range $[0,0.2]$, independently for each component $i$.

From preliminary experiments, we find that stratified sampling $(\gamma=0)$ performs well when $k$ is close to $n / 2$ but does not exhibit good convergence for other $k$ values, with an average error (lack of accuracy) of more than $0.7 \%$ even using 10000 simulations for a 20-component instance (compared to an exact evaluation over all $2^{20}$ scenarios). We also observe that increasing the importance sampling parameter $\gamma$ leads to faster convergence for all $k$ values. The marginal effect diminishes as the value of $\gamma$ increases, i.e., when $\gamma$ changes from 2 to 3 , the accuracy improvement (decrease in average error) is only $0.005 \%$ for a 20 -component instance. Therefore, we choose $\gamma=2$ for the remainder of this text.

\subsection{Tabu parameters}

In this section, we design the best tabu strategies and finetune the parameters. The stopping criterion is 1000 non-improving iterations for each instance, after which the long-term strategies are applied. The complete search procedure ends after the execution of the long-term strategies.

We first look into the number of simulation runs for each instance; we perform these experiments with perfect tests, with move-based tabu classification and a full search of the candidate list. The tabu tenure is chosen as the number $n$ of components. We compute an average optimality gap, as follows: the gap equals $\mid$ Tabu-Best $\mid$ /Best, where Tabu and Best represent the solution returned by the TS procedure and the optimal solution, respectively. The gap is computed with all $2^{20}$ scenarios for each of 10 instances from the 20-component set with $O S=0.6$. The results are shown in Figure 4 . We select 300 simulations (nearly $0.03 \%$ of the total number of scenarios), which leads to $0.8 \%$ average optimality gap within 60 seconds for most of the instances. If the experiments are performed over 5000 simulations, we can acquire solutions with optimality gap around $0.01 \%$ but the computation times are much larger (over 1200 seconds). Our experiments show that the preferable number of simulations is more sensitive to the value of $n$ than to $O S$ and $k$. For the instances with 10 components, the preferable number of simulations is 250 ; the number for 20- 


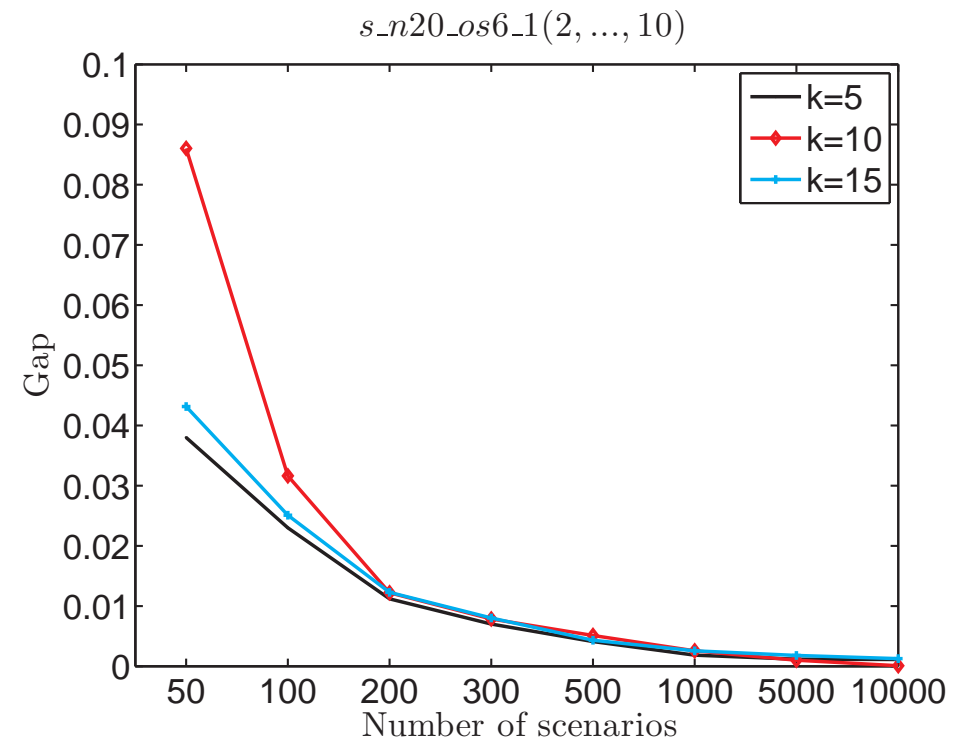

Figure 4: Average optimality gap as a function of the number of simulations

Table 3: Performance comparison between tabu classifications for 20-component instances

\begin{tabular}{r|r|cc}
\hline \multicolumn{2}{|c|}{$O S=0.4$} & Optimality gap & CPU (sec) \\
\hline \multirow{2}{*}{$O S=0.6$} & position-based & $0.84 \%$ & 70.5 \\
& move-based & $0.66 \%$ & 37.2 \\
\hline \multirow{2}{*}{$O S=0.8$} & position-based & $0.68 \%$ & 37.0 \\
& move-based & $0.62 \%$ & 14.6 \\
\cline { 2 - 4 } & position-based & $0.68 \%$ & 8.5 \\
\hline
\end{tabular}

component systems is 300,450 for 40 -component systems, and 700 for 60 -component systems. We conclude that $200+n \sqrt{n}$ turns out to be a reasonable number of simulations for varying $n$. Once the search is completed, the algorithm will return the testing sequence and corresponding expected testing costs. For the sake of accuracy, the algorithm uses $500 \cdot n$ scenarios in this final step to evaluate the solution.

We now compare the performance of the two tabu classification methods. The experiments are performed on the 30 instances of the 20-component set, over a tenure range of $[10,100]$ with increments of 10. The initial solution generation method HR and the SA candidate list strategy are selected. For each order strength, the optimality gap is averaged over 100 experiments. The results are shown in Table 3. The position-based tabu classification is competitive with the move-based tabu classification, but is much faster. Therefore, position-based tabu classification is chosen in the remainder of this work. 
Table 4: Choices for initial solution heuristic and candidate list strategy that have the best performance for each instance set

\begin{tabular}{rrrr}
\hline & $n=20$ & $n=40$ & $n=60$ \\
\hline$O S=0.4$ & $\mathrm{HR}+\mathrm{SA}$ & $\mathrm{HR}+\mathrm{FA}$ & $\mathrm{HR}+\mathrm{FA}$ \\
\hline$O S=0.6$ & $\mathrm{HR}+\mathrm{SA}$ & $\mathrm{HR}+\mathrm{SA} / \mathrm{FA}$ & $\mathrm{HR}+\mathrm{FA}$ \\
\hline$O S=0.8$ & $\mathrm{HP}+\mathrm{FA} / \mathrm{SA}$ & $\mathrm{HR} / \mathrm{HP}+\mathrm{FA}$ & $\mathrm{HR}+\mathrm{FA}$ \\
\hline
\end{tabular}

Secondly, two initial solution generation heuristics (HR, HP) and four candidate list strategies (FA, SA, AC, RH) are tested by running all combinations. In each of these experiments the tenure is set equal to the instance size $n=20$. After eliminating the dominated strategies (with respect to objective function and runtime), the most promising combinations of initial solution heuristic and candidate list strategy are retained for further analysis; these are presented in Table 4. Generally, for large instances with at least 40 components, FA outperforms other candidate list strategies while for smaller instances, it is better to perform a full examination (SA) of the neighbourhood. This is not unlogical: for large instances, SA needs too much computation time to examine the neighbourhood space such that the search may easily be trapped in suboptimal regions, while FA is more favorable towards diversification. For small instances, both FA and SA lead to the best solutions, but SA is slightly faster than FA. When the neighbourhood space is large, both SA and FA become very slow, while AC can speed up the search procedure with an acceptable compromise of runtime and solution quality (see details in Section 6.3). In some cases, HP has the best performance, even though HR generates better initial solutions for most of the instances. We conclude that different algorithmic settings are suitable dependent on the value of $n$.

Thirdly, we need to determine a good tabu tenure value. For this purpose, we have tested the 20-component and 30-component instances with each combination of order strength $O S=0.4,0.6$ and 0.8 and $k=5,10$ and 15 , and tabu tenures over the range $[10,100]$ with increments of 10 . From this extensive experiment we have learnt that no single tenure value can promise a good solution to all problem instances. We therefore apply an empirical formula based on the problem attributes that leads to the best solutions for each instance class. We have found that for instances with higher order strength, relatively smaller tenure values perform better. One reason for this might be that higher order strength significantly reduces the neighbourhood space. Additionally, the tenure value seems to be more sensitive to the instance size and the order strength, and less to the value of $k$. 
As a conclusion, $m \sqrt{n} / O S$ turns out to be a reasonable choice with $m=5$. Furthermore, the tenure formula used along with a candidate list strategy is determined as $\sqrt{n / O S}$. This tabu tenure is applied in all the experiments performed below, unless otherwise noted.

Finally, we calibrate the parameters of diversification and intensification. These two long-term strategies are applied after the pre-specified 1000 non-improving iterations. The diversification strategy steers the search to other directions by multiplying the current tabu tenure by a multiplier for a certain number (e.g., 100) of iterations. A high value for the multiplier enables the algorithm to search very broadly but consumes too much computation time. Based on our experiments, choosing $\sqrt{n}$ turns out to constitute a compromise between solution quality and search effort. The intensification strategy adopted consists of clearing the current memory and conducting a new search on two elite solutions stored in the short-term memory. An elite solution is defined as a best solution that cannot be improved for $\sqrt{n} / O S$ iterations, or $\sqrt{n}$ iterations if a candidate list strategy is used.

\subsection{Performance evaluation of the TS algorithm}

The developed TS algorithm is used to solve the instance sets. We first test the validity of the TS algorithm on the instances under perfect test conditions, and then present the computational results for the same instance sets when the tests are imperfect.

In all the tables below, "opt ${ }_{p}$ " represents the expected cost of the optimal solutions of the instances under perfect test conditions; the optimal olutions are obtained by means of the branchand-bound (B\&B) algorithm of Wei et al. (2013). Entry "opt" refers to the optimal expected cost of the same set of instances under imperfect test conditions with $T=0.9$; an optimal solution with imperfect tests is found by a modification of the B\&B algorithm where the solution is evaluated based on a full enumeration of the scenarios during the search. This will be possible only for $n=10$. "E[E. $[$ " represents the expected cost of the solution produced by the TS algorithm for each of the two settings (perfect and imperfect tests). Accordingly, column "gap ${ }_{p}$ " contains the value $100\left(\mathbb{E}[\cdot]-o p t_{p}\right) / o p t_{p}$, and "gap" indicates the value $100(\mathbb{E}[\cdot]-o p t) / o p t$. The average optimality gap and computation time (CPU) are summarized at the bottom of the tables, where the second line of the CPU time indicates the time after which the best-found solution is not improved any further. Note that the value in each cell is accurate to the second decimal place. The percentage increase 
Table 5: Final results for 10-component instances with $k=5$

\begin{tabular}{|l|rrr|rrr|r|}
\hline & \multicolumn{3}{|c|}{ Perfect tests } & \multicolumn{3}{|c|}{ Imperfect tests } & \\
\hline \hline Instance & opt $_{p}$ & $\mathbb{E}[\cdot]$ & \% gap & opt & $\mathbb{E}[\cdot]$ & \% gap & $\%$ \\
\hline 10o4_1 & 215.07 & 215.07 & 0 & 283.71 & 283.71 & 0 & 31.92 \\
10o4_2 & 234.18 & 234.18 & 0 & 260.16 & 260.16 & 0 & 11.09 \\
10o4_3 & 140.57 & 140.57 & 0 & 170.86 & 170.86 & 0 & 21.55 \\
10o4_4 & 216.20 & 216.20 & 0 & 255.98 & 256.05 & 0.03 & 18.40 \\
10o4_5 & 125.01 & 125.01 & 0 & 142.53 & 142.53 & 0 & 14.01 \\
10o4_6 & 188.63 & 188.63 & 0 & 219.93 & 219.93 & 0 & 16.60 \\
10o4_7 & 241.49 & 241.49 & 0 & 288.34 & 288.34 & 0 & 19.40 \\
10o4_8 & 213.16 & 213.16 & 0 & 253.36 & 253.36 & 0 & 18.86 \\
10o4_9 & 204.79 & 204.87 & 0.04 & 232.41 & 232.41 & 0 & 13.49 \\
10o4_10 & 173.62 & 173.62 & 0 & 212.61 & 212.61 & 0 & 22.46 \\
\hline avg.gap & & & 0 & & & 0.02 & 18.78 \\
avg.CPU (sec) & & 7.43 & & & 9.11 & & \\
& & 0.02 & & & 0.03 & & \\
\hline 10o8_1 & 226.54 & 226.54 & 0 & 260.97 & 260.97 & 0 & 15.20 \\
10o8_2 & 159.31 & 159.31 & 0 & 183.00 & 183.00 & 0 & 14.87 \\
10o8_3 & 232.68 & 232.68 & 0 & 277.01 & 277.01 & 0 & 19.05 \\
10o8_4 & 223.38 & 223.38 & 0 & 257.40 & 257.40 & 0 & 15.23 \\
10o8_5 & 135.46 & 135.46 & 0 & 150.40 & 150.40 & 0 & 11.03 \\
10o8_6 & 207.72 & 207.72 & 0 & 233.59 & 233.59 & 0 & 12.45 \\
10o8_7 & 238.69 & 238.76 & 0.03 & 299.04 & 299.12 & 0.03 & 25.28 \\
10o8_8 & 221.04 & 221.04 & 0 & 269.68 & 269.68 & 0 & 22.01 \\
10o8_9 & 209.64 & 209.64 & 0 & 247.88 & 247.88 & 0 & 18.24 \\
10o8_10 & 190.91 & 190.91 & 0 & 237.79 & 237.79 & 0 & 24.56 \\
\hline avg.gap & & & 0 & & & 0 & 17.79 \\
avg. CPU (sec) & & 2.95 & & & 3.57 & & \\
& & 0.003 & & & 0.002 & & \\
\hline
\end{tabular}

of the optimal testing cost for imperfect case compared with the perfect case is given in the last column $(\%)$.

For the 10-component instance set, both with perfect as well as imperfect test conditions, the TS algorithm finds good solutions within very short runtimes. The results are summarized in Table 5 . Unlike the exact algorithms such as the B\&B algorithm, for which the most time-consuming instances are the ones with low $O S$ and $k \approx n / 2$ (Wei et al., 2013), our TS algorithm is not sensitive to the value of $k$. The reason is that the TS does not use $k$ to calculate a lower bound to eliminate unpromising solutions, and its bottleneck is mainly determined by the size of the solution space.

Also for the 20-component instance set (both with perfect and imperfect test conditions), the TS algorithm performs quite well. The results are presented in Tables $6,7,8$ and 9, for perfect and imperfect testing and different $O S$-values. The dynamic tenure strategy is tested on the 20component instances under perfect test conditions, and we use multiplier values $m=0.5,5$ and 10 

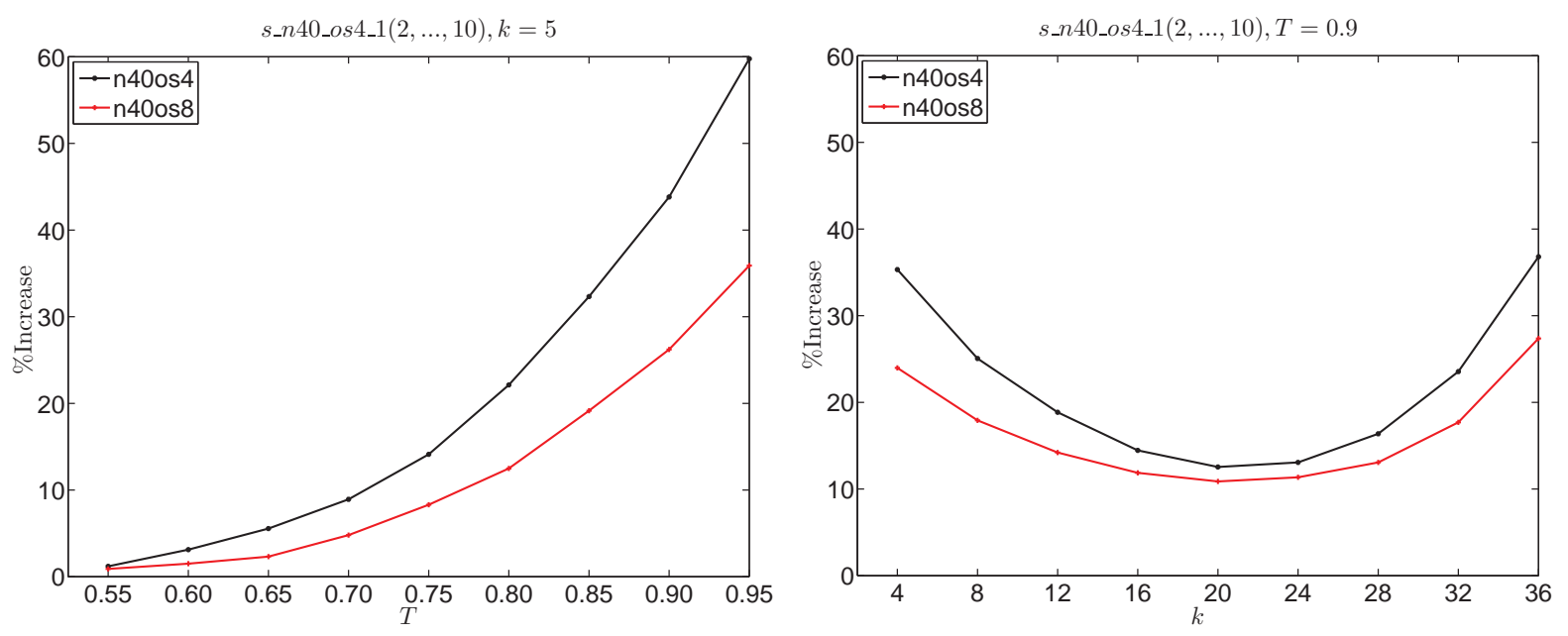

Figure 5: Average percentage increase of the total expected cost, as a function of the threshold (left) and $k$ values (right)

to vary the tenure values. Three sets of tenure values $0.5 \sqrt{n} / O S$ (Low, L), $5 \sqrt{n} / O S$ (Medium, M) and $10 \sqrt{n} / O S$ (High, H) are used and three different tenure strategies, namely LMHM, HMLM and MSML, are applied on each set of instances. Each of the tenure values in a strategy is applied for $\sqrt{n} / O S$ iterations. We observe that low $O S$-values greatly increase the CPU time. For the perfect case, LMHM together with HR + SA outperforms other combinations; the best results are in the fifth and sixth column of Table 6 and 7. Long-term strategies are tested as well. The last four columns of Table 6 and 7 show that the diversification strategy improves the final solution but consumes three times more computation time.

The best strategy combinations validated with perfect tests are subsequently applied to the 20component instances with imperfect tests. We report the results for different threshold values $T$ in Table 8 and 9, together with the increase in expected testing costs compared to perfect test conditions. A similar comparison is graphically depicted in Figure 5 for instances with $n=40$. Logically, the expected costs increase as the threshold value $T$ increases. The importance of the increase varies between different instance settings. Interestingly, this difference decreases as $k$ approaches $n / 2$.

The computational results for large instances with up to 100 components under perfect test conditions are briefly presented in Table 10, where each cell contains the average over 10 instances. We find that the candidate strategy AC2 together with dynamic tenure value pattern LMHM is usually the best both regarding speed as well as solution quality (see the fifth and sixth column of 
Table 6: Final results for 20-component instances with $k=5$ under perfect test conditions $(O S=$ $0.4)$

\begin{tabular}{|c|c|c|c|c|c|c|c|c|c|}
\hline $\begin{array}{l}\text { Tenure } \\
\text { Strategies } \\
\text { Simulations }\end{array}$ & & \multicolumn{2}{|c|}{$\begin{array}{c}5 \sqrt{n} / O S \\
\mathrm{HR}+\mathrm{SA} \\
\\
300\end{array}$} & \multicolumn{2}{|c|}{$\begin{array}{c}\text { LMHM } \\
\text { HR + SA } \\
300\end{array}$} & \multicolumn{2}{|c|}{$\begin{array}{c}5 \sqrt{n} / O S \\
\mathrm{HR}+\mathrm{SA} \\
\text { Diversification } \\
300\end{array}$} & \multicolumn{2}{|c|}{$\begin{array}{c}5 \sqrt{n} / O S \\
\mathrm{HR}+\mathrm{SA} \\
\text { Intensification } \\
300\end{array}$} \\
\hline Instance & $o p t_{p}$ & $\mathbb{E}[\cdot]$ & $\% \operatorname{gap}_{p}$ & $\mathbb{E}[\cdot]$ & $\% \operatorname{gap}_{p}$ & $\mathbb{E}[\cdot]$ & $\% \operatorname{gap}_{p}$ & $\mathbb{E}[\cdot]$ & $\% \operatorname{gap}_{p}$ \\
\hline 20o4_1 & 157.33 & 157.33 & 0 & 157.52 & 0.12 & 157.33 & 0 & 158.01 & 0.43 \\
\hline $20 \mathrm{o} 4 \_2$ & 164.51 & 165.94 & 0.87 & 165.63 & 0.68 & 164.51 & 0 & 165.71 & 0.73 \\
\hline 20o4_3 & 202.20 & 202.20 & 0 & 202.46 & 0.13 & 202.81 & 0.30 & 202.20 & 0 \\
\hline 20o4_4 & 180.32 & 180.99 & 0.37 & 180.62 & 0.17 & 180.46 & 0.08 & 180.66 & 0.19 \\
\hline $2004 \_5$ & 136.07 & 136.89 & 0.60 & 136.07 & 0 & 136.07 & 0 & 137.01 & 0.69 \\
\hline 20o4_6 & 210.70 & 211.71 & 0.48 & 211.71 & 0 & 210.87 & 0.08 & 211.33 & 0.30 \\
\hline 20o4_7 & 171.46 & 171.70 & 0.14 & 172.18 & 0.42 & 171.70 & 0.14 & 172.28 & 0.48 \\
\hline 20o4_8 & 153.22 & 153.03 & 0.53 & 153.65 & 0.28 & 153.59 & 0.24 & 154.00 & 0.51 \\
\hline 20o4_9 & 174.71 & 175.56 & 0.49 & 175.01 & 0.17 & 174.88 & 0.10 & 174.71 & 0 \\
\hline $20 \mathrm{o} 4 \_10$ & 117.79 & 118.52 & 0.62 & 118.30 & 0.43 & 117.89 & 0.09 & 119.32 & 1.32 \\
\hline $\begin{array}{l}\text { avg. gap } \\
\text { avg. CPU }\end{array}$ & & $\begin{array}{r}78.23 \\
0.50\end{array}$ & 0.41 & $\begin{array}{r}61.35 \\
0.78\end{array}$ & 0.24 & $\begin{array}{r}301.22 \\
2.57\end{array}$ & 0.10 & $\begin{array}{r}185.91 \\
1.03\end{array}$ & 0.15 \\
\hline
\end{tabular}

Table 7: Final results for 20-component instances with $k=5$ under perfect test conditions $(O S=$ $0.8)$

\begin{tabular}{|c|c|c|c|c|c|c|c|c|c|}
\hline $\begin{array}{l}\text { Tenure } \\
\text { Strategies } \\
\text { Simulations }\end{array}$ & & \multicolumn{2}{|c|}{$\begin{array}{c}5 \sqrt{n} / O S \\
\mathrm{HR}+\mathrm{SA} \\
\\
300\end{array}$} & \multicolumn{2}{|c|}{$\begin{array}{c}\text { LMHM } \\
\text { HR + SA } \\
300\end{array}$} & \multicolumn{2}{|c|}{$\begin{array}{c}5 \sqrt{n} / O S \\
\text { HR + SA } \\
\text { Diversification } \\
300\end{array}$} & \multicolumn{2}{|c|}{$\begin{array}{c}5 \sqrt{n} / O S \\
\mathrm{HR}+\mathrm{SA} \\
\text { Intensification } \\
300 \\
\end{array}$} \\
\hline Instance & $o p t_{p}$ & $\mathbb{E}[\cdot]$ & $\% g^{\prime} a p_{p}$ & $\mathbb{E}[\cdot]$ & $\% g^{\prime} a p_{p}$ & $\mathbb{E}[\cdot]$ & $\% \operatorname{gap}_{p}$ & $\mathbb{E}[\cdot]$ & $\% g a p_{p}$ \\
\hline 20o8_1 & 269.32 & 269.40 & 0.02 & 269.43 & 0.04 & 270.29 & 0.36 & 269.78 & 0.17 \\
\hline $20 \circ 8 \_2$ & 213.37 & 213.88 & 0.22 & 213.65 & 0.13 & 214.12 & 0.35 & 213.69 & 0.15 \\
\hline 20o8_3 & 301.44 & 301.92 & 0.15 & 301.60 & 0.05 & 301.44 & 0 & 301.44 & 0 \\
\hline 20o8_4 & 288.75 & 289.04 & 0.10 & 289.62 & 0.30 & 289.27 & 0.18 & 289.01 & 0.09 \\
\hline $20 \circ 8 \_5$ & 216.56 & 216.82 & 0.12 & 217.17 & 0.28 & 217.27 & 0.33 & 216.82 & 0.12 \\
\hline 20o8_6 & 255.25 & 256.17 & 0.33 & 255.25 & 0 & 256.07 & 0.32 & 256.07 & 0.32 \\
\hline $20 \circ 8 \_7$ & 230.17 & 230.38 & 0.08 & 230.31 & 0.06 & 230.33 & 0.07 & 230.63 & 0.20 \\
\hline 20o8_8 & 193.83 & 194.20 & 0.18 & 194.06 & 0.12 & 194.984 & 0.52 & 194.12 & 0.15 \\
\hline 20o8_9 & 237.60 & 237.60 & 0 & 237.60 & 0 & 237.86 & 0.11 & 237.96 & 0.15 \\
\hline 20o8_10 & 147.11 & 147.38 & 0.17 & 147.11 & 0 & 147.20 & 0.06 & 147.73 & 0.42 \\
\hline $\begin{array}{l}\text { avg. gap } \\
\text { avg. CPU }\end{array}$ & & $\begin{array}{r}22.43 \\
0.05\end{array}$ & 0.14 & $\begin{array}{r}12.12 \\
0.43\end{array}$ & 0.10 & $\begin{array}{r}64.20 \\
3.20\end{array}$ & 0.23 & $\begin{array}{r}59.12 \\
2.19\end{array}$ & 0.18 \\
\hline
\end{tabular}


Table 8: Final results for 20-component instances with $k=5$ under imperfect test conditions $(O S=$ $0.4)$

\begin{tabular}{|c|c|c|c|c|c|c|c|c|c|c|c|}
\hline $\begin{array}{l}\text { Tenure } \\
\text { Strategies } \\
\text { Simulations }\end{array}$ & & \multicolumn{10}{|c|}{$\begin{array}{c}\sqrt{n} / O S \\
\text { HR + FA } \\
\text { Diversification } \\
200+n \sqrt{n}\end{array}$} \\
\hline$T$ & & \multicolumn{2}{|c|}{0.95} & \multicolumn{2}{|c|}{0.90} & \multicolumn{2}{|c|}{0.85} & \multicolumn{2}{|c|}{0.80} & \multicolumn{2}{|c|}{0.75} \\
\hline Instance & $o p t_{p}$ & $\mathbb{E}[\cdot]$ & $\%$ & $\mathbb{E}[\cdot]$ & $\%$ & $\mathbb{E}[\cdot]$ & $\%$ & $\mathbb{E}[\cdot]$ & $\%$ & $\mathbb{E}[\cdot]$ & $\%$ \\
\hline 20o4_1 & 157.33 & 208.98 & 32.83 & 200.66 & 27.54 & 199.23 & 26.63 & 181.90 & 15.61 & 170.37 & 8.29 \\
\hline 20o4_2 & 164.51 & 229.41 & 39.45 & 205.13 & 24.69 & 202.64 & 23.18 & 193.88 & 17.85 & 183.69 & 11.66 \\
\hline 20o4_3 & 202.20 & 259.41 & 28.29 & 242.09 & 19.73 & 238.33 & 17.87 & 227.56 & 12.54 & 211.90 & 4.80 \\
\hline $2004 \_4$ & 180.32 & 242.99 & 34.76 & 223.28 & 23.82 & 218.05 & 20.92 & 210.85 & 16.93 & 199.51 & 10.64 \\
\hline 20o4_5 & 136.07 & 185.06 & 36.00 & 172.08 & 26.46 & 164.16 & 20.65 & 161.19 & 18.46 & 156.29 & 14.86 \\
\hline $20 \mathrm{o} 4 \_6$ & 210.70 & 269.61 & 27.96 & 252.39 & 19.79 & 243.78 & 15.70 & 237.34 & 12.64 & 228.58 & 8.48 \\
\hline $20 \mathrm{o} 4 \_7$ & 171.46 & 255.19 & 48.83 & 225.46 & 31.49 & 217.92 & 27.10 & 210.89 & 23.00 & 194.13 & 13.22 \\
\hline $2004 \_8$ & 153.22 & 209.36 & 36.64 & 197.00 & 28.58 & 190.82 & 24.54 & 179.78 & 17.34 & 164.18 & 7.15 \\
\hline 20o4_9 & 174.71 & 215.16 & 23.15 & 204.09 & 16.81 & 200.76 & 14.91 & 195.65 & 11.98 & 190.30 & 8.92 \\
\hline 20o4_10 & 117.79 & 170.16 & 44.46 & 154.26 & 30.96 & 146.24 & 24.15 & 143.09 & 21.48 & 135.41 & 14.96 \\
\hline avg. & & & 35.24 & & 24.99 & & 21.57 & & 16.78 & & 10.30 \\
\hline
\end{tabular}

Table 9: Final results for 20-component instances with $k=5$ under imperfect test conditions $(O S=$ $0.8)$

\begin{tabular}{|c|c|c|c|c|c|c|c|c|c|c|c|}
\hline $\begin{array}{l}\text { Tenure } \\
\text { Strategies } \\
\text { Simulations }\end{array}$ & & \multicolumn{10}{|c|}{$\begin{array}{c}\sqrt{n} / O S \\
\text { HR + FA } \\
\text { Diversification } \\
200+n \sqrt{n}\end{array}$} \\
\hline$T$ & & \multicolumn{2}{|c|}{0.95} & \multicolumn{2}{|c|}{0.90} & \multicolumn{2}{|c|}{0.85} & \multicolumn{2}{|c|}{0.80} & \multicolumn{2}{|c|}{0.75} \\
\hline Instance & $\overline{o p t}_{p}$ & $\mathbb{E}[\cdot]$ & $\%$ & $\mathbb{E}[\cdot]$ & $\%$ & $\mathbb{E}[\cdot]$ & $\%$ & $\mathbb{E}[\cdot]$ & $\%$ & $\mathbb{E}[\cdot]$ & $\%$ \\
\hline $20 \mathrm{o} 8 \_1$ & 269.32 & 341.40 & 26.77 & 315.98 & 17.33 & 308.99 & 14.73 & 294.09 & 9.20 & 288.76 & 7.22 \\
\hline $20 \circ 8 \_2$ & 213.37 & 286.31 & 34.19 & 267.26 & 25.26 & 253.63 & 18.87 & 242.60 & 13.70 & 232.58 & 9.00 \\
\hline 20o8_3 & 301.44 & 370.15 & 22.79 & 350.08 & 16.14 & 344.42 & 14.26 & 334.72 & 11.04 & 320.11 & 6.19 \\
\hline $20 \circ 8 \_4$ & 288.75 & 352.90 & 22.22 & 331.69 & 14.87 & 326.21 & 12.97 & 319.93 & 10.80 & 304.35 & 5.40 \\
\hline $20 \circ 8 \_5$ & 216.56 & 275.29 & 27.12 & 256.82 & 18.59 & 250.98 & 15.89 & 238.63 & 10.19 & 232.11 & 7.18 \\
\hline $20 \circ 8 \_6$ & 255.25 & 326.06 & 27.74 & 303.70 & 18.98 & 291.69 & 14.28 & 286.44 & 12.22 & 273.93 & 7.32 \\
\hline $20 \circ 8 \_7$ & 230.17 & 320.74 & 39.35 & 293.06 & 27.32 & 277.09 & 20.39 & 269.79 & 17.21 & 256.07 & 11.25 \\
\hline 20o8_8 & 193.83 & 243.56 & 25.66 & 228.67 & 17.98 & 221.41 & 14.23 & 210.32 & 8.51 & 202.45 & 4.45 \\
\hline 20o8_9 & 237.60 & 272.20 & 14.56 & 266.55 & 12.18 & 261.13 & 9.90 & 249.22 & 4.89 & 249.40 & 4.97 \\
\hline 20o8_10 & 147.11 & 180.66 & 22.80 & 172.42 & 17.21 & 165.06 & 12.20 & 158.03 & 7.42 & 156.74 & 6.55 \\
\hline avg. & & & 26.32 & & 1855 & & 14.77 & & 10.52 & & 6.95 \\
\hline
\end{tabular}


Table 10: Final results for larger instances with $k=5$ under perfect test conditions

\begin{tabular}{|c|c|c|c|c|c|c|c|c|c|}
\hline $\begin{array}{l}\text { Tenure } \\
\text { Strategies } \\
\text { Simulations }\end{array}$ & & \multicolumn{2}{|c|}{$\begin{array}{c}\sqrt{n} / O S \\
\mathrm{HR}+\mathrm{FA} \\
200+n \sqrt{n}\end{array}$} & \multicolumn{2}{|c|}{$\begin{array}{c}\text { LMHM } \\
\text { HR + AC2 } \\
200+n \sqrt{n}\end{array}$} & \multicolumn{2}{|c|}{$\begin{array}{c}\sqrt{n / O S} \\
\text { HR + AC2 } \\
\text { Diversification } \\
200+n \sqrt{n}\end{array}$} & \multicolumn{2}{|c|}{$\begin{array}{c}\sqrt{n / O S} \\
\text { HR }+ \text { AC } \\
\text { Intensification } \\
200+n \sqrt{n}\end{array}$} \\
\hline Instances & $o p t_{p}$ & $\% g a p_{p}$ & CPU & $\% \operatorname{gap}_{p}$ & CPU & $\% \operatorname{gap}_{p}$ & CPU & $\% \operatorname{gap}_{p}$ & CPU \\
\hline $40 \mathrm{o} 4$ & - & 1.34 & $\begin{array}{r}157.40 \\
7.91\end{array}$ & 0.64 & $\begin{array}{r}111.20 \\
4.02\end{array}$ & 0.41 & $\begin{array}{r}578.11 \\
17.31\end{array}$ & 0.80 & $\begin{array}{r}262.32 \\
11.87\end{array}$ \\
\hline $40 \mathrm{o} 8$ & - & 0.29 & $\begin{array}{r}116.54 \\
3.77\end{array}$ & 0.17 & $\begin{array}{r}77.35 \\
2.06\end{array}$ & 0.29 & $\begin{array}{r}382.06 \\
4.92\end{array}$ & 0.36 & $\begin{array}{r}282.29 \\
6.03\end{array}$ \\
\hline $60 \mathrm{o} 4$ & - & 3.45 & $\begin{array}{r}287.87 \\
22.78\end{array}$ & 1.41 & $\begin{array}{r}213.62 \\
18.73\end{array}$ & 0.66 & $\begin{array}{r}498.23 \\
98.12\end{array}$ & 3.01 & $\begin{array}{r}398.34 \\
67.89\end{array}$ \\
\hline $60 \mathrm{o} 8$ & - & 1.88 & $\begin{array}{r}201.35 \\
13.84\end{array}$ & 0.32 & $\begin{array}{r}129.04 \\
9.33\end{array}$ & 0.49 & $\begin{array}{r}411.87 \\
72.82\end{array}$ & 1.25 & $\begin{array}{r}298.77 \\
33.97\end{array}$ \\
\hline 8004 & - & 6.87 & $\begin{array}{r}745.87 \\
55.93\end{array}$ & 3.27 & $\begin{array}{r}633.08 \\
42.43\end{array}$ & 4.39 & $\begin{array}{l}845.34 \\
102.86\end{array}$ & 5.87 & $\begin{array}{r}821.23 \\
82.90\end{array}$ \\
\hline 8008 & - & 3.82 & $\begin{array}{r}487.38 \\
39.84\end{array}$ & 1.39 & $\begin{array}{r}419.20 \\
23.92\end{array}$ & 1.78 & $\begin{array}{r}712.72 \\
60.84\end{array}$ & 3.10 & $\begin{array}{r}542.27 \\
46.03\end{array}$ \\
\hline $100 \mathrm{o} 4$ & - & 9.02 & $\begin{array}{r}1764.12 \\
125.92\end{array}$ & 5.29 & $\begin{array}{r}1361.39 \\
95.39\end{array}$ & 6.72 & $\begin{array}{r}2423.54 \\
224.04\end{array}$ & 8.05 & $\begin{array}{r}2018.44 \\
187.35\end{array}$ \\
\hline $100 \mathrm{o} 8$ & - & 6.48 & $\begin{array}{r}1394.56 \\
95.05\end{array}$ & 4.58 & $\begin{array}{r}955.47 \\
67.38\end{array}$ & 5.42 & $\begin{array}{r}1854.03 \\
193.57\end{array}$ & 6.05 & $\begin{array}{r}1507.30 \\
121.94\end{array}$ \\
\hline
\end{tabular}

Table 10). A full examination (SA) of the neighbourhood for 20-component instances needs only limited runtimes, but for larger instances $(n \geq 40)$ it consumes undesirably large CPU times. Candidate list strategies that restrict the search to a subset of the neighbourhood space are thus applied to the larger instances. As was observed supra in Table 4, FA outperforms other strategies for fixed tabu tenure; the results for FA are in the third and fourth column of Table 10. Long-term strategies have also been tested. For larger instances, the TS procedure using long-term strategies takes much longer before the stop criteria are met (eighth and tenth column), but also improves the final results (seventh and ninth column).

\section{Summary and conclusions}

In this paper, we have studied the complex problem of sequentially diagnosing a $k$-out-of- $n$ system where the component tests are precedence-related and the test of each component has a probability of giving wrong information (so-called "imperfect tests"). This problem is NP-hard even with perfect tests. We have developed a tabu search algorithm that can solve both perfect as well as imperfect testing instances. The major advantage of the tabu search is its capability of identifying 
good solutions within a reasonable time limit; in particular, for small instances it finds optimal or near-optimal instances. The major parameters of the tabu algorithm are determined through extensive computational experiments.

For all but the smallest instances, the evaluation of a single solution based on a full enumeration of the scenario space is intractable, and we therefore sample a number of scenarios using a conditional Monte Carlo simulation technique (importance sampling). The larger the number of scenarios generated, the more accurate the estimate of the objective value becomes, and we try to achieve a reasonable balance between computational effort and solution accuracy. It would be very interesting to find alternative ways for evaluating solutions efficiently and accurately.

Overall, there is no single strategy combination that is dominant for all the testing instances. Generally, for small and medium-size instances it is preferable to use larger numbers of scenarios to reach a high accuracy level, while for large instances it is wiser to spend more effort on a broader search of the solution space. Our computational study shows that applying a dynamic tenure strategy or a diversification strategy with a moderate tenure value is useful in identifying high-quality regions of the solution space. Our experiments also reveal that the expected cost of identifying the state of a complex system can be significantly higher for the imperfect case than for perfect tests, especially when the desired confidence level is high.

\section{Acknowledgements}

This work was supported by the Fundamental Funds for Humanities and Social Sciences of Beijing Jiaotong University under Grant No. 2017jbwy004.

\section{References}

Ahlswede, R., \& Wegener, I. 1987. Search Problems. New York: Wiley.

Altman, D.G., \& Bland, J.M. 1994a. Statistics notes: Diagnostic tests 1: Sensitivity and specificity. British Medical Journal, 308, 1552.

Altman, D.G., \& Bland, J.M. 1994b. Statistics notes: Diagnostic tests 2: Predictive values. British Medical Journal, 309, 102. 
Arostegui, M.A., Kadipasaoglu, S.N., \& Khumawala, B.M. 2006. An empirical comparison of tabu search, simulated annealing, and genetic algorithms for facilities location problems. International Journal of Production Economics, 103(2), 742-754.

Augugliaro, A., Dusonchet, L., \& Sanseverino, E.R. 1999. Genetic, simulated annealing and tabu search algorithms: Three heuristic methods for optimal reconfiguration and compensation of distribution networks. International Transactions on Electrical Energy Systems, 9(1), 35-41.

Ballestín, F., \& Leus, R. 2009. Resource-constrained project scheduling for timely project completion with stochastic activity durations. Production and Operations Management, 18(4), $459-474$.

Bellman, R.E. 1957. Dynamic Programming. Princeton: Princeton University Press.

Ben-Dov, Y. 1981. Optimal testing procedures for special structures of coherent systems. Management Science, 27(12), 1410-1420.

Bilge, Ü., Kiraç, F., Kurtulan, M., \& Pekgün, P. 2004. A tabu search algorithm for parallel machine total tardiness problem. Computer \& Operations Research, 31, 397-414.

Bilge, Ü., Kurtulan, M., \& Kiraç, F. 2007. A tabu search algorithm for the single machine total weighted tardiness problem. European Journal of Operational Research, 176(3), 1423-1435.

Biller, B., \& Ghosh, S. 2006. Multivariate input process. Chap. 5, pages 123-153 of: Handbooks in Operations Research and Management Science: Simulation, vol. 13. Elsevier.

Bratley, P., Fox, B.L., \& Schrage, L.E. 1983. A Guide to Simulation. Springer-Verlag.

Broström, G., \& Nilsson, L. 2000. Acceptance/rejection sampling from the conditional distribution of independent discrete random variables, given their sum. Statistics, 34, 247-257.

Butterworth, R. 1972. Some reliability fault-testing models. Operations Research, 20(2), 335-343.

Cordeau, J.F., Laporte, G., \& Pasin, F. 2008. Iterated tabu search for the car sequencing problem. European Journal of Operational Research, 191(3), 945-956.

Cramer, E., \& Kamps, U. 1996. Sequential order statistics and $k$-out-of- $n$ systems with sequentially adjusted failure rates. Annals of the Institute of Statistical Mathematics, 48(3), 535-549.

De Reyck, B., \& Leus, R. 2008. R\&D-project scheduling when activities may fail. IIE Transactions, 40(4), 367-384.

Glover, F., \& Laguna, M. 1997. Tabu Search. Norwell, MA, USA: Kluwer Academic Publishers. 
Gluss, B. 1959. An optimum policy for detecting a fault in a complex system. Operations Research, 7(4), 468-477.

Graham, R. L., Lawler, E. L., Lenstra, J. K., \& Rinnooy Kan, A. H. 1979. Optimization and approximation in deterministic sequencing and scheduling: A survey. Annals of Discrete Mathematics, 5, 287-326.

Hertz, A., \& Widmer, M. 2003. Guidelines for the use of meta-heuristics in combinatorial optimization. European Journal of Operational Research, 151(2), 247-252.

Huseby, A.B., Naustdal, M., \& Vårli, I.D. 2004. System reliability evaluation using conditional Monte Carlo methods. Tech. rept. Statistical Research Report 2. University of Oslo.

Kelly, F. P. 1982. A remark on search and sequencing problems. Mathematics of Operations Research, 7(1), 154-157.

Kochenberger, G., Glover, F., \& Alidaee, B. 2002. An effective approach for solving the binary assignment problem with side constraints. International Journal of Information Technology \& Decision Making, 1(1), 121-129.

Laguna, Manuel, Barnes, J.Wesley, \& Glover, FredW. 1991. Tabu search methods for a single machine scheduling problem. Journal of Intelligent Manufacturing, 2(2), 63-73.

Law, A.M., \& Kelton, W.D. 2000. Simulation Modeling and Analysis. McGraw-Hill.

Luo, X.G., Kwong, C.K., Tang, J.F., \& Tu, Y.L. 2012. Optimal product positioning with consideration of negative utility effect on consumer choice rule. Decision Support Systems, 54(1), 402-413.

Morton, T., \& Pentico, D.W. 1993. Heuristic scheduling systems: with applications to production systems and project management. Vol. 3. John Wiley \& Sons.

Nachlas, J.A., Loney, S.R., \& Binney, B.A. 1990. Diagnostic-strategy selection for series systems. IEEE Transactions on Reliability, 39(3), 273-280.

Nilsson, L. 1997. On the simulation of conditional distributions in the Bernoulli case. Tech. rept. Research report 14. Department of Mathematical Statistics, Umeå University, Sweden.

Petrovic, D., Duenas, A., \& Petrovic, S. 2007. Decision support tool for multi-objective job shop scheduling problems with linguistically quantified decision functions. Decision Support Systems, 43(4), 1527-1538. 
Pinedo, M. 2012. Scheduling. Theory, Algorithms, and Systems. Springer.

Raghavan, V., Shakeri, M., \& Pattipati, K. 1999. Test sequencing algorithms with unreliable tests. IEEE Transactions on Systems, Man and Cybernetics - Part A: Systems and Humans, 29(4), $347-357$.

Reeves, C.R. 1993. Improving the efficiency of tabu search for machine sequencing problems. Journal of the Operational Research Society, 44(4), 375-382.

Salloum, S. 1979. Optimal testing algorithms for symmetric coherent systems. Ph.D. thesis, University of Southern California.

Schäfer, C., \& Chopin, N. 2013. Sequential Monte Carlo on large binary sampling spaces. Statistics and Computing, 23, 163-184.

Sörensen, K. 2015. Metaheuristics - the metaphor exposed. International Transactions in Operational Research, 22(1), 3-18.

Stork, F. 2001. Stochastic resource-constrained project scheduling. Ph.D. thesis, TU Berlin, Germany.

Ünlüyurt, T. 2004. Sequential testing of complex systems: A review. Discrete Applied Mathematics, 142, 189-205.

Wagner, B.J., \& Davis, D.J. 2001. Discrete sequential search with group activities. Decision Sciences, 32(4), 557-573.

Wan, G.H., \& Yen, B.P.C. 2002. Tabu search for single machine scheduling with distinct due windows and weighted earliness/tardiness penalties. European Journal of Operational Research, 142(2), 271-281.

Wei, W., Coolen, K., \& Leus, R. 2013. Sequential testing policies for complex systems under precedence constraints. Expert Systems with Applications, 40, 611-620.

Wei, W., Coolen, K., Talla Nobibon, F., \& Leus, R. 2017. Minimum-cost diagnostic strategies for $k$-out-of- $n$ systems with imperfect tests. Discrete Applied Mathematics, 222, 185-196. 